\title{
Hauttumoren als Illustration von Charaktereigen- schaften - Beobachtungen in der Gemäldegalerie des Museo di Capodimonte in Neapel
}

R. Möhn

\author{
Skin Tumours as an Illustration of Character Traits - Observations Made in the \\ Picture Gallery of the Museo di Capodimonte in Naples
}

\section{Zusammenfassung}

Bei einem Besuch in der Gemäldegalerie des Museo di Capodimonte in Neapel sind dem Autor drei Darstellungen von vermutlich bösartigen Hauttumoren aufgefallen, nämlich einem Plattenepithelkarzinom bei einem noch jungen Mann auf einem Gemälde von Rosso Fiorentino, einem malignen Melanom auf einem solchen von Velazquez und nochmals einem malignen Tumor bei Lucas Cranach d. Ä. Es wird der Versuch unternommen, die Darstellung dieser Tumoren nicht als Selbstzweck zu begreifen, sondern sie mit den Charaktereigenschaften der dargestellten Personen in Zusammenhang zu bringen, nämlich Unvollkommenheit und Unfertigkeit bei Rosso Fiorentino, Demut und Geduld bei Velazquez, sowie persönlicher Bösartigkeit bei Lucas Cranach.
Abstract

During a visit of the picture gallery of the Museo di Capodimonte in Naples the author discovered cases of apparently malignant skin tumours in three paintings. The first one is a squamous cell carcinoma of a young man in a painting by Rosso Fiorentino, the second is a malignant melanoma in a picture by Velazquez and a third one is another malignant tumour in a painting by Lucas Cranach the Elder. The author tries to demonstrate that the presentation of those tumours is not an end in itself, but can be seen as an expression of the character traits of the persons presented in the pictures, namely imperfection and immaturity with Rosso Fiorentino, humbleness and patience with Velazquez or personal malice with Lucas Cranach.

\section{Einleitung}

Der Palazzo Reale di Capodimonte, hoch über Neapel in einem weitläufigen Park gelegen, war anfangs des 18. Jahrhunderts zunächst nur als kleines Jagdschlösschen geplant. Nachdem aber der Bourbone Karl III. die Herrschaft über die Stadt übernommen hatte und die unermesslichen, schon von Papst Paul III. Farnese begründeten Kunstsammlungen des als Herzog von Parma 1731 ohne Nachkommen verstorbenen Antonio Farnese erbte, musste zwischen 1738 und 1755 ein viel größerer, zum Museum erweiterter Bau errichtet werden, um diese alle aufnehmen zu können. So hat sich im Laufe der Jahrhunderte eine einzigartige Kunstsammlung entwickelt. Vertreten sind nicht nur erstrangige Gemälde, sondern auch Skulpturen, Porzellan-, Glasarbeiten und Kunsthandwerk. Seit 1947 gehört alles dem italienischen Staat, und seit 1957 sind die Sammlungen als Museum zugänglich [1]. Umfangreiche Restaurierungen machten es mit seiner herrlichen
Lage im Grünen, dem gewaltigen Bau mit den mächtigen Tonnengewölben des Innenhofes und seiner einzigartigen Atmosphäre, die sowohl residenzartigen als auch musealen Charakter ausstrahlt, zu einem der schönsten Museen, die ich kenne.

In der Gemäldesammlung sind natürlich hauptsächlich italienische Meister aus dem Mittelalter, der Renaissance, dem Manierismus und Barock vertreten. Masaccio, Raffael, Tizian und Caravaggio sind mit hervorragenden Werken repräsentiert.

\section{Plattenepithelkarzinome bei Rosso Fiorentino?}

Als Hautarzt sind mir besonders drei Gemälde aufgefallen. Das erste ist von Rosso Fiorentino, eigentlich Giovanni Battista di Jacopo di Guasparte (Ritratto di giovane, Bildnis eines jungen Mannes). Der Künstler wurde 1494 in Florenz geboren und starb 1540

Korrespondenzadresse

Dr. med. Rudolf Möhn · Hautarzt und Allergologe · Philippsruher Allee 35 • 63454 Hanau

E-mail: RMoehn@aol.com

Bibliografie

Akt Dermatol 2006; 32: 148-151 (c) Georg Thieme Verlag KG Stuttgart · New York

DOI $10.1055 / \mathrm{s}-2005-921162 \cdot$ ISSN 0340-2541 
in Paris. Mit Pontormo wurde er zum Begründer des Florentiner Manierismus. Bereits „seine frühen Bilder zeigen den Willen zu einer rational erarbeiteten Komposition straff durchgezeichneter menschlicher Figuren und eine virtuos verwirklichte Vorliebe für zart abgestimmte Farben. ... Besondere Anregungen empfing er durch Michelangelo, Andrea del Sarto und Fra Bartolomeo." [2]. Seine wichtigsten Stationen waren Florenz, Rom, viele andere italienische Städte und ab 1530 Paris, wohin ihn König Franz I als Hofmaler berufen hatte. In dessen Auftrag schuf er von 1534-1537 in der Galerie des Schlosses Fontainebleau sein Hauptwerk, einen Freskenzyklus mit 12 Hauptbildern. In Frankreich ist er als Maître Roux bekannt [3]. Unser Bild (Abb.1), das früher auch dem vielleicht berühmteren Parmigianino zugeschrieben wurde, stammt aus der römischen Zeit und zeigt einen jungen Mann vor einem Alkoven mit ungemachtem Bett und Säulen mit männlichem Kapitell. Stolz und doch lässig entspannt sitzt er auf einem typischen Orientteppich mit bizarrem Muster zum Zeichen eines unreifen Stutzertums. Das Bild, entstanden möglicherweise erst kurz vor dem berüchtigten „sacco di Roma“ 1527, der verheerenden Plünderung Roms durch die Söldnertruppen Karls V., vor denen Rosso Fiorentino flüchtete, ist offenbar nicht ganz vollendet [4], insbesondere die Partien an der rechten Hand und der angrenzenden Hose. Auch die linke Hand macht einen merkwürdigen Eindruck, ist aber nach meiner Meinung nicht unvollendet, sondern zeigt dermatologische Auffälligkeiten (Abb. 2). Bei den lang und spitz ausgezogenen „Madonnenfingern“, die künstlerisch als ein Zeichen des sich langsam entwickelnden Manierismus aufgefasst werden können, sind die Endglieder und Nagelpartien wenig ausgearbeitet und zeigen einen deutlichen Pigmentverlust. Am Kleinfinger ist dieser im Bereich der zweiten und dritten Phalanx besonders deutlich, während die Grundphalanx eher hyperpigmentiert ist. Am dritten und vierten Finger scheinen die proximalen Interphalangealgelenke etwas aufgetrieben. Besonders auffallend aber zwei ulzerierende Tumoren auf dem Handrücken zwischen 2. und 4. Finger. Da bei genauem Hinsehen auch an der Nasenspitze - im zweifellos vollendeten Gesicht - eine krustig bedeckte, wahrscheinlich ebenfalls tumoröse Hautläsion besteht, glaube ich, dass hier mit Absicht eine Person mit Hauttumoren, am ehesten Plattenepithelkarzinomen dargestellt wurde. Wären nur im Gesicht mehr Pigmentflecken zu sehen - einige sind schon da! könnte man in Anbetracht der jugendlichen Person und der Poikilodermie der linken Hand sogar ganz entfernt an ein Xeroderma pigmentosum mit Tumoren an belichteten Stellen vielleicht in einer weniger bösartigen Variante denken. So bleibt die Dignität unklar, auch chronisch entzündliche Veränderungen sind natürlich möglich, die Darstellung solcher Hautveränderungen aber gerade bei einem noch jungen Menschen bemerkenswert.

\section{Auffällige Pigmentläsion in einem Gemälde von Velazquez}

Die zweite Beobachtung betrifft ein Gemälde des berühmten spanischen Malers Velazquez (Diego Rodriguez de Silva y Velazquez), geboren 1599 in Sevilla, gestorben 1660 in Madrid. Das in Madrider Privatbesitz befindliche und sonst wahrscheinlich nur selten in der Öffentlichkeit ausgestellte Bild war in Neapel gerade im Rahmen einer Sonderausstellung mit Werken von Velazquez zu sehen. Es kann nicht der Sinn dieser Arbeit sein, über Leben und Werk dieses sehr bedeutenden Künstlers und Hofmalers

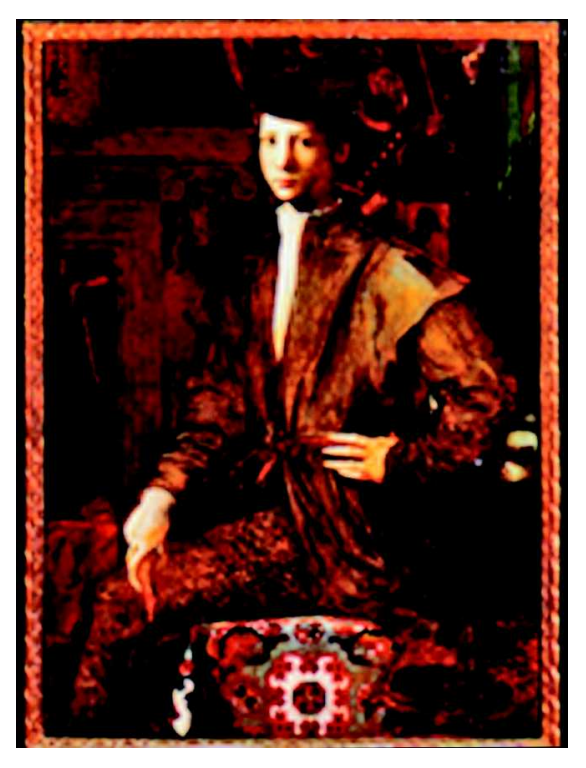

Abb. 1 Rosso Fiorentino, Bildnis eines jungen Mannes: An der Nasenspitze krustig bedeckte Hautveränderungen und an der linken Hand ulzerierende tumoröse Veränderungen.

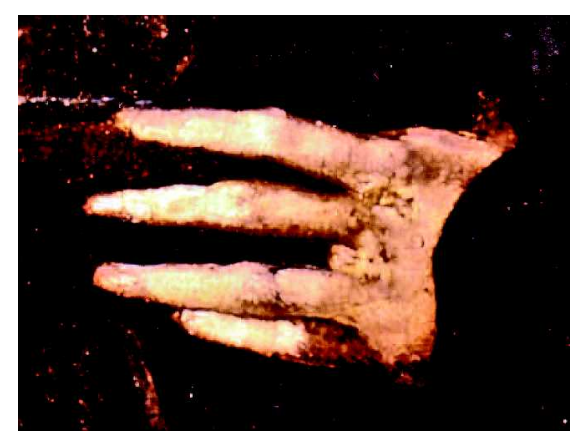

Abb. 2 Rosso Fiorentino, Bildnis eines jungen Mannes: Detail der linken Hand. Man sieht deutlich die ulzerierenden Veränderungen, daneben auch Pigmentinkontinenz, teilweise aufgetriebene Gelenke und „Madonnenfinger".

des spanischen Königs Philipp IV. auch nur annähernd genau zu informieren. Auf die entsprechenden Fachbücher sei daher verwiesen.

Unser Bild (Abb. 3 u. 4), übrigens erst im Jahre 1999 eindeutig dem Schaffen von Velazquez zugeordnet [5], zeigt den Heiligen „San Simone de Rojas“ auf dem Totenbett (geb. zu Valladolid am 28.10.1552, gest. zu Madrid 29. 9.1624), 1735 selig und 1988 heilig gesprochen, von 1573-1580 Studium der Theologie in Salamanca, von 1581 - 1587 Lehrer der Theologie im Ordenskolleg in Toledo, danach Übernahme verschiedener Aufgaben in mehreren Ordenshäusern Kastiliens, von 1621-1624 Provinzial-Beichtvater und Berater König Philipps III. und Erzieher Philipps IV. Er war in Madrid eine außerordentlich einflussreiche religiöse Persönlichkeit, engagierte sich in den dortigen Armenvierteln, förderte die Marienfrömmigkeit und begründete 1611 die Laienbrüderschaft „Congregación de los siervos del dulcísimo Nombre de Maria“ und die „Ave Maria-Congregación“ [6,7]. Künstlerisch besteht bei dem Bild enge Verwandtschaft zu den ebenfalls in der Zeit von 1622-1624 entstandenen Porträts Philipps IV. und des Conte Duca di San Paolo. Valdivieso [8] ordnet „San Simone“ dem im ebenso lebens- wie todessüchtigen Barockzeitalter außerordentlich beliebten Genre der „Vanitas“-Kunst zu, welche die Vergänglichkeit und Nichtigkeit des Menschen und gerade auch der höher gestellten Fürsten und Kleriker zum Thema hat.

Das von links kommende Licht fällt auf ein sorgfältig modelliertes Gesicht mit dem Ausdruck größter Ruhe und abgeklärten Friedens. Dem Dermatologen sticht natürlich die Pigmentläsion 


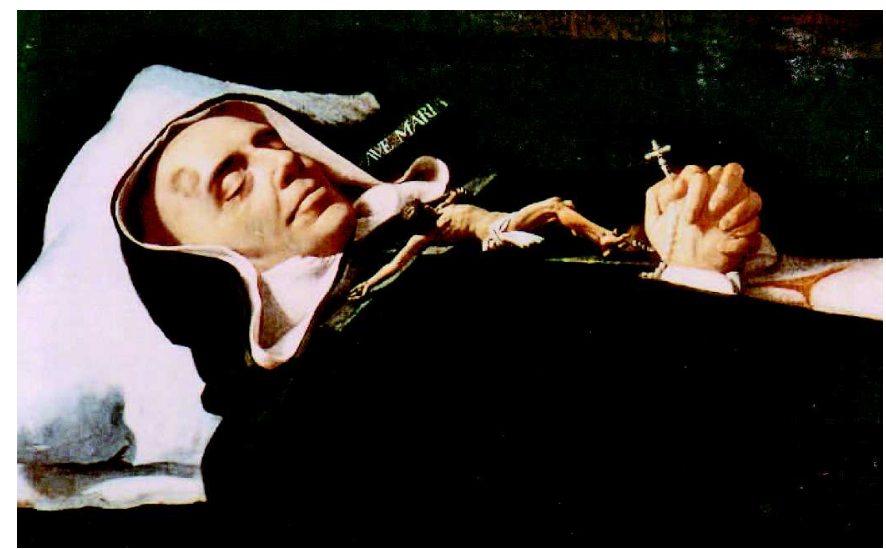

Abb. 3 Diego Velazquez, San Simone de Rojas auf dem Totenbett: Vanitasbild im Genre der Barockzeit. Deutlich erkennbar eine ausgeprägte Pigmentläsion auf der rechten Stirnhälfte, die am ehesten an ein malignes Melanom denken lässt.

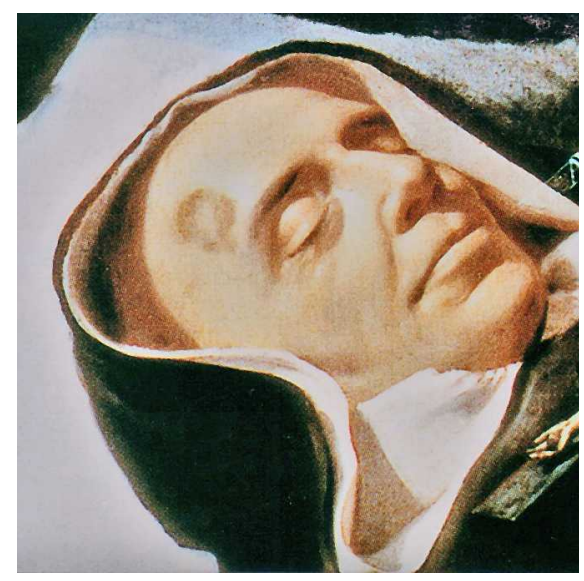

Abb. 4 Diego Velazquez, San Simone de Rojas auf dem Totenbett: Detail der Kopfpartie. Die Pigmentläsion ist hier noch besser erkennbar.

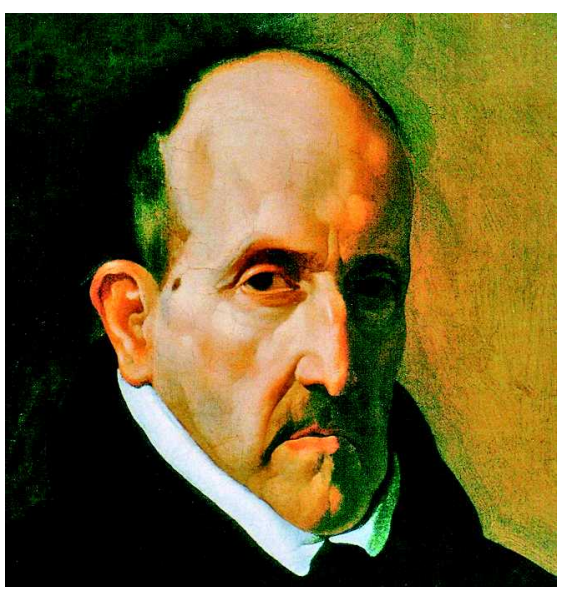

Abb. 5 Diego Velazquez, Bildnis des Luis de Gongora y Argote: Kleine, sicher gutartige Pigmentläsion auf der seitlichen Stirnpartie rechts. Ausgeprägte Vitiligo im Gesicht. Enge stilistische Verwandtschaft zu Abb. 4

auf der rechten Stirnhälfte sofort ins Auge. Um eine zentrale Abblassung sieht man eine im Wesentlichen konzentrisch kreisförmige, an manchen Stellen aber auch nach innen und außen durchaus unregelmäßig ausgefranste Hyperpigmentierung. Nicht genau erkennbar ist, ob es sich nur um eine makulöse, oder um eine flach erhabene Veränderung handelt. Jedoch besteht für mich kein Zweifel, dass hier in erster Linie ein malignes Melanom vom Typ Lentigo maligna oder ein superfiziell spreitendes Melanom mit charakteristischen zentralen Pigmentre- gressionen und unregelmäßiger Begrenzung in Erwägung gezogen werden muss. Andere gutartige Läsionen wie Naevi aller Art, benigne Lentigines und seborrhoische Keratosen zeigen doch in aller Regel ein sehr viel gleichförmigeres Bild. Leider ist es mir bis jetzt nicht gelungen, Informationen über die Todesursache dieser wirklich existent gewesenen Person zu bekommen, sie könnte ja mit der Pigmentläsion in Zusammenhang stehen.

Das Bild ist künstlerisch eng verwandt mit dem ebenfalls in der Sonderausstellung zu sehenden Porträt des Luis de Gongora y $\mathrm{Ar}-$ gote (1622). Dieses zeigt eine gleichförmige, wesentlich kleinere, wahrscheinlich gutartige Pigmentläsion temporal rechts, dafür aber eine deutliche Vitiligo im ganzen Gesicht (Abb. 5).

\section{Gesichtstumor als Zeichen bösartigen Charakters bei Lucas Cranach}

Die dritte Beobachtung betrifft eines der wenigen nicht italienischen Gemälde der Sammlung, nämlich „Christus und die Ehebrecherin“ von Lucas Cranach d. Ä. (geb. 1472 in Kronach, gest. 1553 in Wittenberg). Auch hier wird auf nähere Ausführungen zu Leben und Werk des Künstlers verzichtet und auf die Fachliteratur verwiesen. Der Verfasser hatte Gelegenheit im „Hautarzt“ schon einmal über den Maler und eines seiner Werke zu berichten, deshalb sei die entsprechende Arbeit zitiert, in der ebenfalls nähere Ausführungen zu Leben und Werk des Künstlers zu finden sind [9]. Insbesondere wurde dort darauf hingewiesen, dass Lucas Cranach in seinem weiteren Beruf als Apotheker sicher auch über medizinische Kenntnisse verfügte, was für unser jetzt zu besprechendes Bild von einiger Bedeutung ist.

Die darauf (Abb. 6a) dargestellten Köpfe wirken ein wenig hölzern und bieder, so dass anzunehmen ist, dass es ein Werkstattbild ist, wie so oft bei Cranach. Die Szenerie bezieht sich auf Johannes 8, wo erzählt wird, dass eine soeben ertappte Ehebrecherin von den Pharisäern Christus in der Erwartung zugeführt wird, dass dieser eine schlimme Bestrafung, in der Regel die Steinigung empfehle. Wie allgemein bekannt, tut er dieses aber nicht, sondern ermahnt die Sünderin und appelliert an alle, wie auch am oberen Bildrand schriftlich vermerkt „Wer under Euch on Sünde ist - Der wirft den ersten Stein auf Sie." Das scheint bei den meisten ein Umdenken bewirkt zu haben, so dass man von einer Bestrafung absieht.

Das Gemälde ist ein Beispiel dafür, dass medizinische Betrachtungen darüber nicht etwa wenig sinnvolle Spekulationen sind, sondern von erheblicher Bedeutung für die Interpretation des Kunstwerkes insgesamt sein können. Auffällig sind die Figuren des geharnischten, eher kleinen und untersetzten Mannes links und die des etwas größeren Mannes mit Hammer rechts. Der Kleinere links (Abb. 6b) hält noch die zur Steinigung vorgesehenen Felsbrocken vorrätig. Er hat auf der rechten Wange einen riesigen halbkugelig vorgewölbten, warzenartigen, zum Teil noch behaarten, an den unscharf begrenzten Rändern aber schon enthaarten Tumor, so dass bereits eine bösartige Entwicklung angenommen werden kann, am ehesten ein Plattenepithelkarzinom. Die hässliche Geschwulst, auch wenn man sie im medizinischen Sinn nicht als bösartig ansehen will, steht pars pro toto für die durch den übrigen Habitus charakterisierte Bösartigkeit und Pri- 


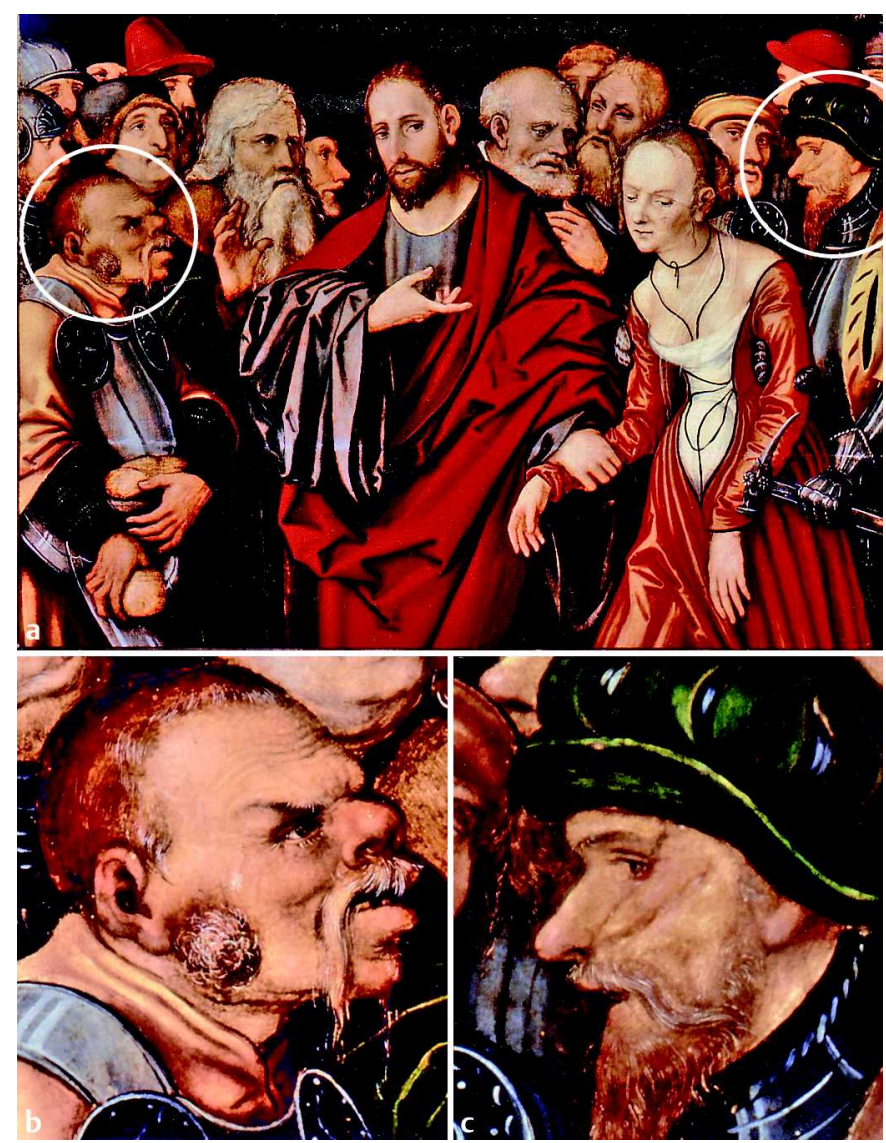

Abb. 6 a Lucas Cranach der Ältere, Christus und die Ehebrecherin. Deutlich ausgeprägter Tumor am rechten Kieferwinkel bei dem Geharnischten links, am ehesten Plattenepithelkarzinom als Zeichen persönlicher Bösartigkeit. b Detail vom Kopf des Geharnischten links. Der Tumor ist hier noch besser erkennbar. c Detail vom Kopf des Geharnischten rechts. Die Narbe wird als symbolisches Zeichen der Reinigung vom Tumor der Bösartigkeit verstanden.

mitivität des Mannes überhaupt. Am liebsten würde er sofort mit der Bestrafung beginnen. Der Geharnischte rechts ist offenbar ein Vertreter der öffentlichen Gewalt. Er hat zwar die Sünderin fest im Griff, wirkt aber bei der Aktion seltsam unbeteiligt und eher lustlos. Er hat im Gesicht und in der Region des linken Auges eine sehr lange auffällige v-förmige Narbe (Abb. 6c). Ist es übertrieben, wenn ich diese Narbe, die sogar ein wenig an einen $\mathrm{Zu}$ stand nach Verschiebeplastik erinnert (zu Zeiten Cranachs durchaus hier und da schon praktiziert!), symbolisch gesehen bereits als ein Zeichen der Befreiung vom Tumor der Bösartigkeit und damit an ein durch Christus bewirktes geläutertes Umdenken interpretiere? Jedenfalls fällt es mir schwer, diese deutliche
Narbe als rein zufällig oder nur als Relikt bei einem alten Haudegen aufzufassen. Auch der schwere Hammer, den er anstatt eines sonst üblichen Degens oder Schwertes trägt, weist diesen Mann als Antipoden des Bösartigen auf der linken Seite aus, ist er doch damit in der Lage, ihm die Steine zu zerschlagen und ihn unschädlich zu machen.

\section{Hauttumoren zur Kennzeichnung von Charaktereigenschaften}

Liegt jedenfalls in diesem Werk Cranachs die Gleichsetzung von bösartigem Hauttumor mit Bösartigkeit der Person klar auf der Hand, fällt es auch bei den anderen beiden Kunstwerken mit der Darstellung von Hauttumoren schwer, nicht an die bei den alten Meistern weit verbreiteten allegorischen und symbolistischen Anspielungen zu denken. Auch hier könnten die Tumoren als ein Mittel zum Ausdruck von Charaktereigenschaften gemeint sein. Bei dem Bild Rosso Fiorentinos erinnern die Hauttumoren in Anbetracht der Entstehungszeit des Bildes kurz vor der Flucht des Künstlers aus Rom und zusammen mit der Jugend der Person, der Poikilodermie, dem bizarren Orientteppich und dem ungemachten Bett an die Darstellung von Unfertigkeit und Unvollkommenheit, während im Bild von Velazquez die friedvollen Gesichtszüge des heiligen San Simone auf Eigenschaften wie Gelassenheit, Geduld, Demut, Frömmigkeit und Tapferkeit beim Ertragen einer schweren auferlegten Krankheit und- selbst im Falle von Gutartigkeit - eines „Makels“ im wahrsten Sinne des Wortes hinweisen.

\section{Literatur}

${ }^{1}$ Höcker C. Golf von Neapel und Kampanien. Köln: Dumont-Kunstreiseführer, 2004: 122

2 Plagemann V. Rosso Fiorentino. dtv-Kindlers Malerei-Lexikon, Band 10. München: 1976: 338

${ }^{3}$ Thieme U, Becker F. Allgemeines Lexikon der bildenden Künstler von der Antike bis zur Gegenwart. Band 29. München: DTV, 1992: 61

${ }^{4}$ Spinosa N (Hrsg). National Museum of Capodimonte. English Ed. Electa Napoli, 1999: 56

${ }^{5}$ Pérez Sánchez AE. Novedades velazqueñas. Archivo Español de Arte, 1999: 288

${ }^{6}$ Sapio M (ed). Velazquez a Capodimonte. Electa Napoli, 2005: 80

${ }^{7}$ Kasper W (Hrsg). Lexikon für Theologie und Kirche IX. Freiburg Herder, 2000: 604

${ }^{8}$ Valdivieso E. Vanidades y desenguños en la pintura española del Siglo de Oro. Fundación Instituto de Espresa, (ohne Jahresangabe): 81 - 83

${ }^{9}$ Möhn R. Medizinische Betrachtungen am Cranach-Altar der St. Wolfgangskirche zu Schneeberg/Erzgebirge. Hautarzt 2002; 53: 622 - 624 